\title{
Study on the Online Monitoring System and Fault Diagnosis Technology of Intelligent Circuit Breaker
}

\author{
Li Yi ${ }^{1, a}$, Jiang Hao ${ }^{1, b}$, Gong Fugao ${ }^{1, c}$, Xia Hengyi $i^{1, d}$ \\ ${ }^{1}$ State Grid Chongqing Electric Power Co. Maintenance Branch Company, Chongqing 400039, \\ China \\ aemail: liyijxgs@163.com, bemail:695921336@qq.com, cemail:1639975572@qq.com, \\ demail:1049731389@qq.com
}

Keywords: Intelligent circuit breaker, Fault diagnosis, Virtual instrument technology, Reliability

\begin{abstract}
Intelligent circuit breaker is one of the most significant devices in the electric system, and its stability and reliability are quite significant for the operation of the power grid. An online monitoring and fault diagnosis system based on the vibration signal of virtual instrument technology is proposed according to the analysis of online monitoring of vibration signal in intelligent circuit breaker. The system employs the LabVIEW software, for programming HMI and accomplishing the storage, display, analysis and treatment, etc. of the measured data. The sensor and digital signal processing unit forms the field monitoring module and it is installed on the breaker for accomplishing the acquisition and transmission of mechanical parameters, breakingclosing current signal and vibration signal. The experimental results show that the system can reflect the mechanical operating state of intelligent circuit breaker in real time, with friendly manmachine interaction, complete functions and high reliability, etc. Moreover, it implements the expected system of the online monitoring system.
\end{abstract}

\section{Introduction}

The rapid development of the electric system, especially the construction of intelligent substation, sets much stricter requirements on the reliability of high voltage electrical apparatus[1,2]. Breaker, as insulation and arc extinction device, plays the role of control and protection of the power grid. When it breaks down, it may result in great economic loss and social impact. Consequently, whether it can reinforce the online monitoring of high voltage intelligent circuit breaker is related to the safe operation of the entire electric system. According to the statistical analysis result of CIGRE, about $80 \%$ of faults in breaker are mechanical failure[3,4]. Therefore, online monitoring of the mechanical features of high voltage intelligent circuit breaker, early discovery of potential equipment fault, and comprehensive monitoring of all state indexes of the breaker will be favorable for reducing the fault rate and improving the safety and reliability of electric system[5].

There are domestic and foreign studies on all state characteristics of intelligent breaker and most are about the mechanical vibration performance[6]. America, Norway, Australia, Japan, etc. have already determined some practical vibration signal processing method till now. A. D. Stokes, et al from Australia proposed the index damped oscillation model and extracted the vibration signal with a mathematical model. A. A. Polycarpou et al. put forward an analysis method based on the enveloping analysis of vibration signal, and Norway Electric Power Research Institute proposed the DTW method[7]. Domestic institutions of higher learning, such as Tsinghua University, Shandong University, etc. put forward a method of processing the vibration signal by establishing the mathematical model. Since the mechanical vibration signal of breaker is also quite complicated, a mechanical fault may contain abundant vibration signal, and the fault type reflected by distinct vibration signals is not unique. Domestic and foreign research methods mainly focus on the processing of vibration signal, and lack the contemporary acquisition and processing of several characteristic signals[8].

Based on the complexity of mechanical failure of breaker and diversity of characteristic signal, a kind of online monitoring and fault diagnosis system based on the virtual instrument technology and 
LabVIEW software by analyzing the monitoring principles of several main characteristic signals of the breaker (for instance the journey - time characteristic of breaker, switching-closing coil current), which may provide a new plan or the fault detection of intelligent breaker.

\section{Principles of online monitoring}

The opening-closing brake of breaker is controlled by the electromagnetic force of the circuit[9]. Consequently, the failure monitoring of the breaker can be implemented by analyzing the current change of the control coil of opening-closing brake and motion characteristics of moving contact.

The current waveform of the opening-closing brake control coil directly reflects the dynamics of iron core motion, for instance, the tripping, block, etc. The failure-free current waveform is shown in Fig. 1, where t0 - $\mathrm{t} 1$ iron core is in static state. During $\mathrm{t} 2$, the iron core starts moving. It can evaluate if there are abnormal conditions in the movement process of iron core by installing the current sensor on the breaker and detecting the current changes of control coil.

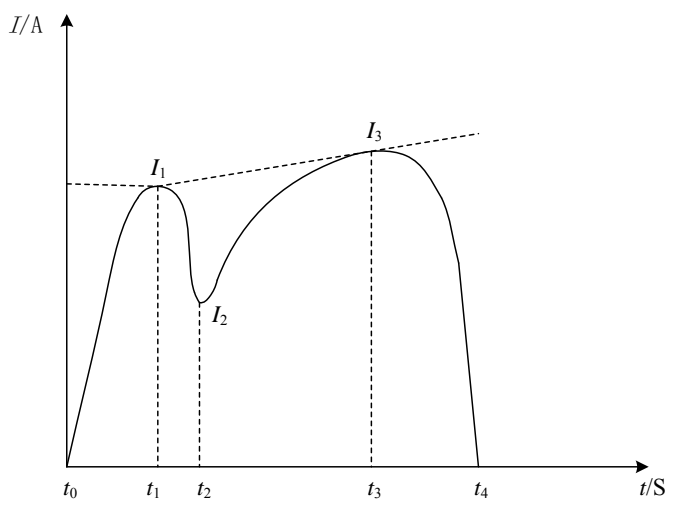

Fig. 1 Normal current waveform of the opening-closing brake of the breaker

The relationship between the motion displacement rules of the moving contact in the intelligent circuit breaker and the motion displacement rules of the main coupling lever is approximated as the characteristic of straight line, while the time response curve of the contact travel time may reflect if there are faults if there are fault in the mechanical vibration. The failure-free travel time characteristic curve is shown in Fig. 2, and it can gain the motion characteristic signals of the contact by installing the angular displacement sensor, linear position sensor, and acceleration sensor, so as to judge if the intelligent circuit breaker has abnormal conditions[10].

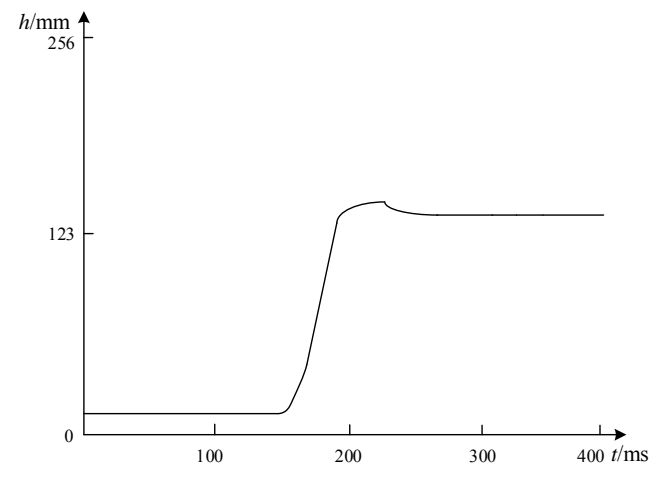

Fig. 2 Characteristics curve of the travel time of breaker contact

\section{System structure and hardware design of the intelligent breaker}

In this paper, multi-sensor based online monitoring system of intelligent breaker is designed according to the monitoring principles of the mechanical failure of intelligent breaker, and the structure is shown in Fig. 3. The system integrate the field test unit based on the sensor module and DSP data processing unit, as well as the human-computer interface and data storage central control unit based on the virtual instrument technology[11]. 
The sensor would collect the real-time data of the breaker motions and send the data to DSP for processing, and the processed data would be sent to the central control unit of the monitoring system[12]. The control unit mainly finishes the storage, treatment, display and fault diagnosis of measured data, etc. The human-computer interface mainly compiles with the LabVIEW software, and carries out the communication between the data acquisition units through CAN bus.

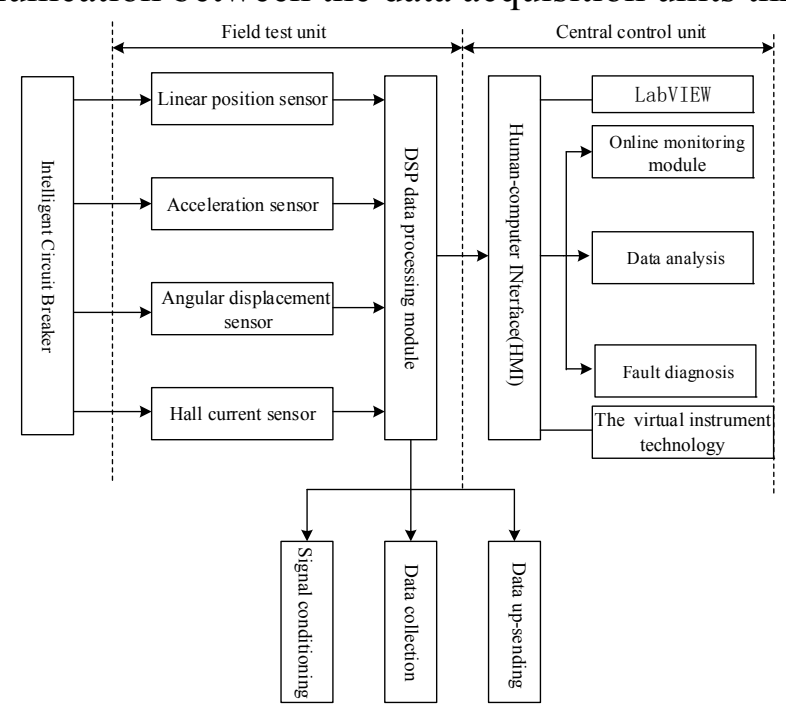

Fig. 3 Overall structure of the online monitoring system of intelligent breaker

The breaker is installed with the linear position sensor (model WDL-25-2), acceleration sensor (LC0166T), and angular displacement sensor (model WDD35D-4) for measuring the travel-time characteristic curve of the mechanical movement, and Hall current sensor (YDG-HSD) for measuring the current signal of the opening-closing brake. Finally, all sensors are integrated into the data acquisition module.

The human-computer interface detection system platform based on the virtual instrument technology covers four parts, including the data acquisition, HMI, fault diagnosis and analysis and data storage. The data acquisition mainly implements the collection of mechanical characteristic parameters of the breaker through the PCI-1714UI driving programs; HMI implements the manmachine interaction, display the data monitoring result, and realize the visual interface of all functions; fault diagnostic analysis plays the role of background central control, and is responsible for accepting the characteristic signal of breaker from HMI, analyzing data, connecting all function modules, and playing the role of data transmission. The characteristic signal data acquired by PCI1714 UI can be stored with the database or text, for the post-stage analysis and inspection.

The intelligent breaker monitoring system software is mainly developed with the virtual instrument LabView, and its HMI mainly implements the initialization setting, real-time display of channel signal, channel selection, triggering setting, data storage, etc. Compared with the traditional detection instrument, the system combines the traditional instrument hardware and computer, expands the functions of traditional instrument, and accomplishes the data acquisition, analysis and display.

Since the breaker characteristic signals collected by the acquisition card are huge in amount, a database is created with the database access technology and LabSQL in order to gain signals correctly, so as to manage the complicated monitoring tasks and storing the monitoring data, which implements the visits and management between the LabView and AQLServer database, and solves the defects of LabView in the access and management of database effectively. LabSQL mainly packages a series of LabSQL Vis by taking advantage of the Microsoft ADO and SQL operation. It can have access to the SQLServer database and Microsoft Access database, and the system object base employs the Microsoft Access database.

When the closing and opening control loop of the breaker is connected, the data can be acquired by triggering the sensor in the closing and opening coil. The system mainly carries out the fault diagnosis for the mechanical characteristics signal of the breaker by making use of the time domain 
analysis method in Express VI. In practical application, it mainly acquires data from the sensor, and there would be a certain gap from the data acquisition card after the expansion of conditioner. Considering the impact of all aspects of factors, the mechanical characteristic signals acquired should be pre-treated, and then the signal data consistent with the sensor measuring the frequency spectrum can be obtained through the band-pass filter. In order to prevent the distortion of waveform, it must satisfy the Shannon's sampling theorem, namely the sampling frequency is two times higher than the highest frequency of the analysis signal.

\section{Test results}

The software of monitoring system mainly realizes the following functions: CAN bus operation and data transmission, display of the current waveform of closing-opening brake, and display of travel-time waveform of the contact motion. Through establishing the simulation experiment platform, simulating the actual mechanical motions of the breaker, and detecting the vibration signal of breaker, the waveform of the experiment acquisition data is shown in Fig. 4.

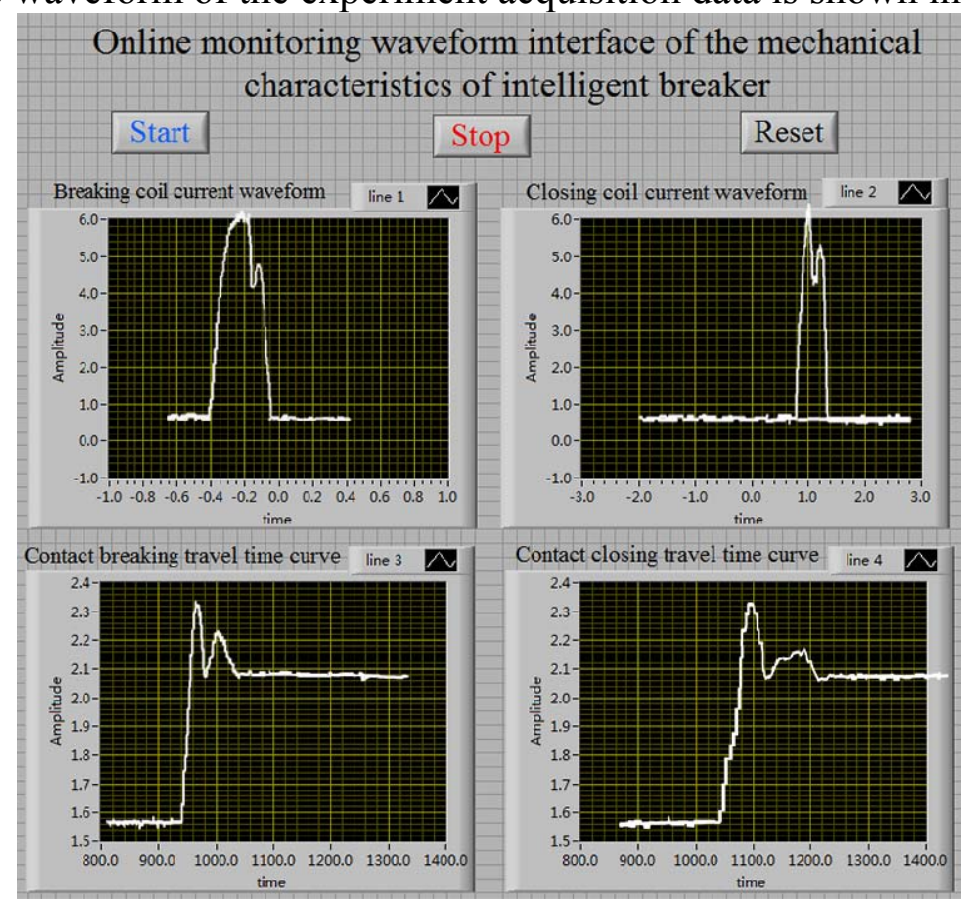

Fig. 4 Online monitoring waveform interface of the mechanical characteristics of intelligent breaker

After acquiring the waveform of opening-closing coil current and waveform of contact traveltime, the mechanical vibrational state of the breaker and if there would be faults in corresponding time nodes can be analyzed according to the change analysis of waveform. In table 1, it is the waveform time parameter calculation result of three groups of coil current waveform, which can reflect the mechanical vibrational state of the breaker.

Table 1 Time parameter calculation result of the coil current waveform

\begin{tabular}{|c|c|c|c|c|c|}
\hline Measured parameters & $\mathrm{t} 0$ & $\mathrm{t} 1$ & $\mathrm{t} 2$ & $\mathrm{t} 3$ & $\mathrm{t} 4$ \\
\hline Coil current waveform 1 & 0.0 & 23.5 & 34.5 & 51.5 & 58 \\
\hline Coil current waveform 2 & 0.0 & 24.0 & 35.0 & 52.0 & 56.5 \\
\hline Coil current waveform 3 & 0.0 & 25.0 & 35.5 & 51.0 & 56.0 \\
\hline
\end{tabular}




\section{Conclusion}

The development of smart power grids sets higher demands on the reliability of intelligent circuit breaker, and as for the systematic monitoring of running status and timely discovery of faults, it should also be much more intelligent, automatic and systematic. In this paper, the online monitoring system of intelligent breaker is established by combining virtual instrument technology and LabVIEW software, and it can implement the collection of opening-closing brake coil current and travel-time characteristic signals through the field data acquisition unit. Besides, it can display the measured waveform in real time with the data processing $d$ analysis module of human-computer interface, and evaluate the mechanical vibration fault according to the variation conditions.

The establishment of experiment platform for simulation experiment shows that the system can collect signals effectively, which would provide reliable waveform parameters for the fault diagnosis and implements the fault detection of intelligent breaker.

\section{Acknowledgment}

In this paper, the research was sponsored by the Nature Science Foundation of China (Project No. 51207175).

\section{References}

[1] Huang Haibo, Tao Chen, Liu Bin, Online Monitoring Technology and State Evaluation of the Mechanical Characteristics of the Intelligent Breaker [J]. High Voltage Apparatus, 2015, (03): 129$134+139$.

[2] Zhou Yongbin, Design and Development of Intelligent Low-voltage Breaker based on the Smart Power Grid [J]. Electrical \& Electronics, 2015, (02): 62-64.

[3] Chang Guang, Study on the High-voltage Circuit-breaker Vibration Monitoring and Fault Diagnosis [D]. Beijing Jiaotong University, 2013.

[4] Ning Nan, 220kV and above split-phase breaker auxiliary switch two times on different periods [J]. Water Conservancy \& Electric Power Machinery, 2013, (06): 47-49+53+86.

[5] Duan Xu, Research on Transformer Vibration Mechanical and Signal Analysis under DC Bias [D]. Chongqing University, 2013.

[6] Wang Yubo, Study on the Performance Analysis and Optimization of Operating Mechanism in Intelligent Circuit Breaker [D]. Hebei University of Technology, 2012.

[7] Huang Xinbo, Wang Xiaokuan, Fang Shouxian, He Xia, Xiao Yuan, Design on the Status Monitoring IED of Circuit Breaker for Smart Substation [J]. Automation of Electric Power Systems, 2012, (22): 95-99.

[8] Chang Guang, Zhang Zhenqian, Wangyi, Review of Vibration Diagnosis of Mechanical Fault of High Voltage Circuit Breaker [J]. High-Voltage Electrical Appliances, 2011, (08): 85-90.

[9] Zeng Qingjun, Lin Yanxia, Development and Fault Diagnosis of Intelligent On-line Monitoring System of High Voltage Vacuum Circuit Breaker[J]. Journal of Southeast University (Natural Science Edition), 2009,(S2):42-48.

[10] Dong Yue, SF_6 Analysis on the On-line Monitoring and Vibration Signal of High Voltage Circuit Breaker[D]. Shanghai Jiaotong University, 2008.

[11] Ding Wenjun, Research and Development of Condition Monitoring System of High Voltage Circuit Breaker [D]. Zhejiang University,2006. 
[12] Liu Xiaoming, High Voltage SF_6 Circuit Breaker Arc Dynamic Mathematical Model and Optimization Design of Nozzle Structure[D]. Shenyang University of Technology, 2003. 